\title{
Collective Labor Supply with Children
}

\author{
Richard Blundell \\ University College London and Institute for Fiscal Studies \\ Pierre-André Chiappori \\ University of Chicago and Columbia University \\ Costas Meghir
}

University College London and Institute for Fiscal Studies

\begin{abstract}
We extend the collective model of household behavior to allow for the existence of public consumption. We show how this model allows the analysis of welfare consequences of policies aimed at changing the distribution of power within the household. Our setting provides a conceptual framework for addressing issues linked to the "targeting" of specific benefits or taxes. We also show that the observation of the labor supplies and the household demand for the public good allow one to identify individual welfare and the decision process. This requires either a separability assumption or the presence of a distribution factor.
\end{abstract}

\section{Introduction}

The "targeting" view.-It is by now widely accepted that intrahousehold distribution of income and decision power matters. Numerous empirical

This research is part of the program of research of the Economic and Social Research Council Centre for the Microeconomic Analysis of Public Policy at IFS. We thank Ben Pollak, four anonymous referees, and seminar participants at the University of St. Louis and IFS for their useful comments. We are especially indebted to the editor (Fernando Alvarez) for his unusually detailed and insightful comments. The usual disclaimer applies.

[Journal of Political Economy, 2005, vol. 113, no. 6]

(C) 2005 by The University of Chicago. All rights reserved. 0022-3808/2005/11306-0004\$10.00 
studies have shown that, contrary to an implicit postulate of the standard framework, targeting a benefit to a particular household member (say, the wife) may have important consequences on the ultimate use of the corresponding resources. Thomas (1990) argued early on that male and female nonlabor incomes have a very different impact on children's health and demographics; similar conclusions have been reached by, among others, Schultz (1990), Browning et al. (1994), Lundberg, Pollak, and Wales (1997), and Phipps and Burton (1998). More recently, Thomas, Contreras, and Frankenberg (1997), using an Indonesian survey, have shown that the distribution of wealth by gender at marriage has a significant impact on children's health in those areas in which wealth remains under the contributor's control. ${ }^{1}$ Duflo (2003) has derived related conclusions from a careful analysis of a reform of the South African social pension program, which extended the benefits to a large, previously not covered, black population. Specifically, Duflo finds that the consequences of this windfall gain on child nutrition dramatically depend on the gender of the recipient. ${ }^{2}$ Such findings have potentially crucial normative implications on the design of aid policies, social benefits, taxes, and other aspects of public policy.

However, while the "targeting" view has strong empirical support and major policy implications, its theoretical foundations remain somewhat weak. After all, the standard methodological tool for studying household behavior is (or was until recently) the "unitary" model, which relies on the assumption that the household maximizes a unique utility function. This assumption directly implies that targeting cannot be effective, since the various sources of income will always be pooled at the household level for the sake of decision making.

An alternative, increasingly popular, framework for studying household behavior is the "collective" approach, whereby individuals with specific (and in general different) preferences make Pareto-efficient decisions. This conceptual framework includes, as particular cases, bargaining models (under symmetric information) as well as a number of other settings. The collective model is aimed at formalizing the notion of "decision powers" within the household and the idea that changes in respective powers typically generate changes in behavior even when total resources are kept constant-a key insight of the "targeting" view. In that sense, the collective approach seems to provide the natural theoretical background for issues related to targeting.

The first goal of the present paper is to support this claim. We provide a fully developed theoretical framework within which these issues can

\footnotetext{
${ }^{1}$ See also Galasso (1999) for a similar investigation.

${ }^{2}$ See also Rubalcava and Thomas (2000) for a study of the impact of benefits on female labor supply in the United States.
} 
be addressed. In this model the household budget constraint is pooled and there are no "hypothecated" funds. However, complex decision processes are allowed for, provided that they satisfy a basic efficiency requirement; namely, outcomes are assumed to be efficient in the Pareto sense. Different decision processes lead to different locations of the outcomes on the Pareto frontier, and a set of Pareto weights for the family members correspond (implicitly or explicitly) to each location. These weights fully summarize the decision process. A basic goal of the collective approach is precisely to analyze how the decision process, that is, the individual weights, can be affected by prices, incomes, and other exogenous factors and how such changes influence household decisions. From a collective perspective, targeting may thus matter through its impact on respective weights of household members. Paying a benefit to the wife instead of the husband may twist the balance of power in her favor; everything (i.e., preferences and budget constraints) equal, this may (and in general will) ultimately change the outcome.

We illustrate how the collective approach works by considering a particular problem. Several studies argue that, on average, "mothers care more for children than fathers," in the sense that an increase in the mother's power within the couple results in more expenditures made for children. We analyze the theoretical underpinnings of this claim within a collective approach. We prove that a shift in the (Pareto) weights favoring a member boosts the demand for a public good if and only if the marginal willingness to pay of this member is more sensitive to increases in (her) private consumption than that of the other member. In other words, the key property is not that the mother has a larger willingness to pay for child goods (out of her resources), but that her willingness to pay is more responsive to changes in these resources than the father's.

Mapping theory and observed behavior: identifiability.-Our first claim, thus, is that the collective model may provide an adequate conceptual toolbox for analyzing policy issues linked to intrahousehold allocation of power. Still, its empirical relevance has to be asserted. Two aspects are of particular importance, namely, the empirical characterization of household behavior stemming from a collective framework and the identifiability of the structural model from observed behavior.

During the last decade, important progress has been made on the characterization problem. Chiappori (1988, 1992), Browning and Chiappori (1998), and recently Chiappori and Ekeland (2003) have derived increasingly general necessary conditions for a given function to be the collective demand of a group of a given size. However, whenever welfare implications are at stake, characterization is not enough. When theory is used to formulate normative judgments, identifiability becomes a crucial issue. To be useful, a theory should provide ways of recovering the underlying welfare-relevant structure (preferences, de- 
cision process) from observed behavior. From this perspective, the collective model exhibits an important weakness: While some identification results have been obtained so far, basically all rely on the assumption that commodities are privately consumed. ${ }^{3}$ Such a setting, which excludes public consumption within the household, seems inappropriate for the study of decisions regarding children, since it is natural to assume that both parents derive utility (albeit possibly to a different extent) from children's well-being. In other words, while the collective process seems to provide a very natural conceptual context for analyzing intrahousehold allocation of power, it is fair to say that, in the present state, not enough is known about collective behavior with public goods to actually ground empirical analysis within the collective framework.

The second goal of the present paper is precisely to fill this gap. In a related and complementary paper, Chiappori and Ekeland (2004) analyze identifiability in the collective framework from a very general perspective. ${ }^{4}$ In this paper, we concentrate on a version of the collective model that has been extensively used for empirical applications (see, e.g., Fortin and Lacroix 1997; Blundell et al. 2001; Chiappori, Fortin, and Lacroix 2002), namely, Chiappori's (1992) model of collective labor supply, and we introduce children in this specific context. We assume that both parents care about children's welfare (or, equivalently, that expenditures on children are a public good within the household), but possibly to a different extent; identifying from observed behavior how much each parent cares for expenditure on children is precisely one of the goals of this line of research. Using a model that incorporates labor supply is particularly appropriate in the context in which the public expenditures are taken to be for children; in a more general setting analyzed in our paper, time may be used for market activities, household production, and leisure.

We show that this model is fully identifiable; that is, parents' individual preferences and the decision process (as summarized by the Pareto weights) can generically be recovered from observed behavior. ${ }^{5}$ In par-

\footnotetext{
${ }^{3}$ An important exception is a paper by Fong and Zhang (2001), who consider a model in which leisure can be consumed both privately and publicly. Although the two alternative uses are not independently observed, they can in general be identified under a separability restriction, provided that the consumption of another exclusive good (e.g., clothing) is observed.

${ }^{4}$ For further results on the collective model and identification, see also Donni (2000). For an early discussion of children in the context of the collective model, see Bourguignon (1999).

${ }^{5}$ We use here the standard distinction between identifiability and identification (see, e.g., Koopmans 1949). A model is identifiable when an assumed perfect observation of behavior would enable one to fully recover the underlying structural model. For instance, the standard consumer model is identifiable because (as is well known) preferences can be uniquely recovered from demand functions. Identification, on the other hand, relates to the more general problem of the relationship between data and theory, in which the
} 
ticular, the observation of changes in expenditures on children in response to changes in wages allows one to recover each parent's willingness to pay for expenditures on children. As always, identifiability requires restrictions. In our case we show that if leisure and individual consumption are separable from expenditure on children, then we can identify individual preferences on private and public goods as well as the Pareto weights, just by observing individual labor supply, aggregate household consumption, the expenditure on the public good, and wages. We also show that if separability is not a valid assumption, knowledge of a distribution factor, that is, a variable driving the Pareto weights but not preferences, ${ }^{6}$ allows full identification. Interestingly, the separability assumption may be tested without fully identifying the model, allowing us to ascertain the informational requirements for identification a priori.

Finally, the model is extended to include household production. In a way this relaxes further the assumptions of the model because it allows some aspect of time to be public within the household and further reinforces the justification of considering the identification issues within the context of labor supply. We consider a framework in which the public good is used, together with time for each individual, as an input to household production. Again we show that the model is identifiable, under the assumption of productive efficiency, as long as time use data are available detailing the time inputs that go into household production (as opposed to pure leisure). Of course the measurement problems here can be severe, but this discussion points to both the importance of collecting data on time use and the importance of distinguishing expenditure on private and public goods.

\section{The Framework}

\section{A. Commodities, Preferences, and Distribution Factors}

We consider a static version of the collective model of labor supply for a two-member $(i=1,2)$ household. There are three commodities: two individual leisures $L^{1}$ and $L^{2}$ and a Hicksian composite good $C$; wages (respectively nonlabor income) are denoted $w_{1}$ and $w_{2}$ (respectively $Y)$; the price of the Hicksian good is normalized to one. In contrast to

limits to the observation of behavior are paramount. See Chiappori and Ekeland (2004) for a more precise discussion of this distinction and Blundell et al. (2001) for a discussion of identification in a collective framework.

${ }^{6}$ For instance, of variables affecting each member's threat point in a bargaining framework. 
previous versions of the model, we assume that the Hicksian good is used for private expenditures and some public consumption:

$$
C=C^{1}+C^{2}+K
$$

where $K$ denotes the level of expenditures for the public good. A natural (but not exclusive) interpretation is that $K$ represents the amount spent on children. The identifiability results we shall present do not require variations in the price of the public good.

An important tool to achieve identification is the presence of distribution factors (Bourguignon, Browning, and Chiappori 1995). They are defined as variables that can affect group behavior only through their impact on the decision process. Think, for instance, of the choices as resulting from a bargaining process. Typically, the outcomes will depend on the members' respective bargaining positions, and any variable in the household's environment that may influence these positions (extrahousehold environmental parameters in McElroy's [1990] terminology) potentially affects the outcome. Such effects are of course paramount, and their relevance is not restricted to bargaining in any particular sense. One crucial insight of the "targeting" literature is precisely that any variable that changes the balance of power within the household (say, paying a benefit to the wife instead of to the husband), everything equal, may have an impact on observed collective behavior. Throughout the paper, we denote by $z$ a distribution factor, and we assume that $z$ is a continuous variable. $^{7}$

As is standard, we assume that $L^{1}, L^{2}, C$, and $K$ are observed (as functions of $w_{1}, w_{2}, Y$, and $z$ ), whereas the distribution of private consumption within the couple $\left(C=C^{1}+C^{2}\right)$ is not. We assume that the functions $L^{1}\left(w_{1}, w_{2}, Y, z\right), L^{2}\left(w_{1}, w_{2}, Y, z\right), C\left(w_{1}, w_{2}, Y, z\right)$, and $K\left(w_{1}\right.$, $\left.w_{2}, Y, z\right)$ are twice continuously differentiable.

Individual $i$ is characterized by differentiable, strictly increasing, strictly convex preferences $\succsim^{i}$ on leisure, private consumption, and the level of public expenditures. We assume that the bundle $\left(w_{1}, w_{2}, Y, z\right)$ varies within a compact subset $\mathcal{K}$ of $\mathbb{R}_{+}^{3} \times R$; then the vector $\left(L^{i}, C^{i}\right.$, $K$ ) varies within some compact set $\mathcal{K}^{\prime}$ of $\mathbb{R}_{+}^{3}$. The preferences are represented by a twice continuously differentiable utility function $U^{i}$, and we assume that $U^{i}$ is such that the Gaussian curvature of any indifference curve is positive at any point of $K^{\prime}\left(U^{i}\right.$ has no critical point on $\left.\mathcal{K}^{\prime}\right)$. Then preferences can be represented by a differentiable, strictly concave func-

${ }^{7}$ For instance, Chiappori et al. (2002) use sex ratio as a continuous distribution factor for the identification of a collective model of labor supply. Other examples include nonlabor income (Thomas 1990), labor income in a model involving constrained labor supply (Browning et al. 1994), wealth at marriage (Thomas et al. 1997), and benefits (Rubalcava and Thomas 2000). 
tion $U^{i}$ on $\mathcal{K}^{\prime}$. Throughout the paper, we use such a representation of individual preferences.

In some sections of the paper, we make the assumption that individual consumption and leisure are separable from the public good $K$ :

$$
U^{i}\left(L^{i}, C^{i}, K\right)=W^{i}\left[u^{i}\left(L^{i}, C^{i}\right), K\right],
$$

where $W^{i}$ and $u^{i}$ are twice continuously differentiable, strictly increasing, and strongly concave. The separability assumption is certainly restrictive since the level of expenditures on children (say, paying for day care) may be expected to affect the trade-off between consumption and labor supply at the individual level; we discuss below when it can be relaxed. Note, however, that we never assume that individual preferences are not affected by the presence of children. ${ }^{8}$

\section{B. The Decision Process}

Pareto efficiency.-Following the usual strategy of collective models (Chiappori 1988, 1992; Blundell et al. 2001), we assume that the decisions made by the household are Pareto-efficient. This is equivalent to assuming that household allocations are determined as solutions to the problem

$$
\begin{aligned}
\max _{L^{1}, L^{2}, C^{1}, C^{2}, K} H\left(L^{1}, L^{2}, C, K ; \lambda\right)= & \lambda U^{1}\left(L^{1}, C^{1}, K\right) \\
& +(1-\lambda) U^{2}\left(L^{2}, C^{2}, K\right)
\end{aligned}
$$

subject to the overall budget constraint

$$
w_{1} L^{1}+w_{2} L^{2}+C^{1}+C^{2}+K=w_{1}+w_{2}+Y
$$

(where the time endowment is normalized to one). The Pareto weight $\lambda \geq 0$ reflects the relative weight of member 1 in the household. This can be a function of wages $\left(w_{1}\right.$ and $\left.w_{2}\right)$ as well as of nonlabor income $Y$ and distribution factors. We assume that the Pareto weight is continuously differentiable in $w_{1}, w_{2}, Y$, and the distribution factors $z$ (if any). Note that the "household utility function" $H$ is not a standard utility function because it depends on the Pareto weight $\lambda$, which itself varies with prices and income; technically, $H$ is thus price-dependent, which implies, for instance, that the resulting demand functions will typically

\footnotetext{
${ }^{8}$ We do not use (or suggest using) observations on the behavior of couples without children or on singles for identification purposes; in particular, preferences may be different in arbitrary ways for individuals in all these states. The separability concerns expenditures on the public good, taken here to be children. Hence we allow the marginal rate of substitution (MRS) between consumption and leisure to depend on the presence of children, but we assume that it is not significantly affected by how much is actually spent on them.
} 
not satisfy Slutsky symmetry. However, $H$ is a strictly concave function of $\left(L^{1}, L^{2}, C, K\right)$ and exhibits separability properties, in the sense that some MRSs do not depend on the Pareto weight-a property that has crucial implications for the model. Indeed, first-order conditions imply that

$$
\lambda \frac{\partial U^{1}}{\partial C}=(1-\lambda) \frac{\partial U^{2}}{\partial C}
$$

and, from the envelope theorem,

$$
\frac{\partial H}{\partial L^{1}}=\lambda \frac{\partial U^{1}}{\partial L^{1}}, \quad \frac{\partial H}{\partial L^{2}}=(1-\lambda) \frac{\partial U^{2}}{\partial L^{2}}, \quad \frac{\partial H}{\partial C}=(1-\lambda) \frac{\partial U^{2}}{\partial C} .
$$

It follows that

$$
\frac{\partial H / \partial L^{i}}{\partial H / \partial C}=\frac{\partial U^{i} / \partial L^{i}}{\partial U^{i} / \partial C}
$$

In words, at the equilibrium point, the household's MRS between individual $i$ 's leisure and private consumption is equal to the corresponding individual's MRS between her labor supply and consumption. Hence, in general, it depends only on $L^{i}, C^{i}$, and $K$, and not on the spouse's labor supply or on the Pareto weight.

Conditional sharing rule.-Just as in the private consumption case, the solution to the household problem (2) can be thought of as a two-stage process. At stage 1, agents agree on public expenditures as well as on a particular distribution of the residual nonlabor income between them. At stage 2, the two members each freely choose their level of consumption and labor supply, conditional on the level of public expenditures and the budget constraint stemming from stage 1. Technically, let $L^{* i}\left(w_{1}, w_{2}, Y, z\right), C^{* i}\left(w_{1}, w_{2}, Y, z\right), i=1,2$, and $K^{*}\left(w_{1}, w_{2}, Y, z\right)$ be the solution of problem (2), and define $\rho_{i}$ by

$$
\rho_{i}\left(w_{1}, w_{2}, Y, z\right)=w_{i} L^{* i}\left(w_{1}, w_{2}, Y, z\right)+C^{* i}\left(w_{1}, w_{2}, Y, z\right)-w_{i} .
$$

Here, $\rho_{1}$ and $\rho_{2}$ define the conditional sharing rule. In words, $\rho_{i}$ is the fraction of residual nonlabor income allocated to member $i$; $\rho_{i}$ is conditional in the sense that the members share what is left for private consumption after purchasing the public good. Hence $\rho_{i}$ can be positive or negative, and

$$
\rho_{1}\left(w_{1}, w_{2}, Y, z\right)+\rho_{2}\left(w_{1}, w_{2}, Y, z\right)=Y-K^{*}\left(w_{1}, w_{2}, Y, z\right) .
$$


Clearly, with $K=K^{*}\left(w_{1}, w_{2}, Y, z\right)$ taken as given, the functions $L^{* i}\left(w_{1}\right.$, $\left.w_{2}, Y, z\right)$ and $C^{* i}\left(w_{1}, w_{2}, Y, z\right)$ solve

$$
\begin{aligned}
& \max _{L^{i}, C^{i}} U^{i}\left(L^{i}, C^{i}, K\right) \\
& \text { subject to } w_{i} L^{i}+C^{i}=w_{i}+\rho_{i}\left(w_{1}, w_{2}, Y, z\right) ;
\end{aligned}
$$

that is, agents each choose their private consumption and labor supply by maximizing utility, under the constraint that they cannot spend more than their share of residual nonlabor income. ${ }^{9}$

An important remark is that in contrast to the private-good case, the existence of a (conditional) sharing rule, while implied by efficiency, is not equivalent to efficiency: an arbitrary sharing rule is not in general compatible with efficiency for a given level of public expenditures. The intuition for this result goes as follows. For given preferences, there exists a continuum of Pareto-efficient allocations. In general, ${ }^{10}$ different efficient outcomes correspond to different levels of the public good and different distributions of private consumptions and labor supplies. Hence, although, conditional on $K$, a consumption/labor supply allocation may be (constrained) efficient, the particular level $K$ may fail to solve an efficient household allocation problem under the prevailing prices. This shows the limit of the "two-stage" interpretation of the decision process; while formally convenient (as will become clear below), it should not hide the fact that the level of public expenditures cannot be chosen independently of the allocation of private resources.

From a more technical perspective, for any given sharing rule, compatibility with the observed level of public expenditures imposes additional conditions, a crucial point for the identifiability results below. However, before we state these conditions, it is convenient to introduce two concepts of indirect utility.

Indirect utilities.-Let $V^{i}\left(w_{i}, \rho_{i}, K\right)$ denote the value of program (4); intuitively, $V^{i}$ is a conditional (on $K$ ) indirect utility function for $i$. We call it the individual indirect utility of agent $i$ because it depends only on $i$ 's preferences; that is, it does not vary with the particular decision process at stake (although its argument $\rho_{i}$ certainly does). As usual, there is a one-to-one correspondence between direct and indirect utilities; that is, $U^{i}$ can be deduced from $V^{i}$ by

$$
\begin{aligned}
& U^{i}\left(L^{i}, C^{i}, K\right)=\min _{w_{i} \rho_{i}} V^{i}\left(w_{i}, \rho_{i}, K\right) \\
& \text { subject to } w_{i} L^{i}+C^{i}=w_{i}+\rho_{i} .
\end{aligned}
$$

\footnotetext{
${ }^{9}$ If a higher utility could be achieved at the same cost (say, for some $\left(L^{\prime i}, C^{\prime i}\right)$ ), then the maximand in (2) could be increased by replacing $\left(L^{i}, C^{i}\right)$ with $\left(L^{\prime i}, C^{\prime i}\right)$, a contradiction.

${ }^{10}$ That is, except for very particular cases such as quasi-linear preferences.
} 
Note also that since preferences are strictly increasing, $\partial V^{i} / \partial \rho_{i}>0$, at each point.

Second, for any particular function $\rho_{i}\left(w_{1}, w_{2}, Y, z\right)$, we can express $V^{i}$ directly as a function of wages, nonlabor income, and public expenditures. This requires a slightly technical construction, however, since we must formally translate the fact that $K$ is kept constant. Specifically, let $\mathcal{O}$ be some open subset of $\mathcal{K}$ such that $\partial K^{*} / \partial z$ does not vanish on $\mathcal{O}$. By the implicit function theorem, the condition $K^{*}\left(w_{1}, w_{2}, Y\right.$, $z)=K$ allows one to express $z$ as some function $\zeta$ of $\left(w_{1}, w_{2}, Y, K\right)$. Then we can define, over $\mathcal{O}$, the function $\tilde{V}^{i}$ by

$$
\tilde{V}^{i}\left(w_{1}, w_{2}, Y, K\right)=V^{i}\left[w_{i}, \rho_{i}\left(w_{1}, w_{2}, Y, \zeta\left(w_{1}, w_{2}, Y, K\right)\right), K\right] .
$$

In words, $V^{i}$ describes $i$ 's indirect utility when facing a private allocation $\rho_{i}$ and a level of expenditures on children $K$, whereas for any given function $\rho_{i}, \tilde{V}^{i}$ describes $i$ 's indirect utility when faced with a wage income bundle $w_{1}, w_{2}, Y$, and a distribution factor $z$ such that public expenditures are exactly $K$. The distribution factor plays a key role here because it provides an additional dimension, thus allowing $w_{1}, w_{2}$, and $Y$ to vary freely (i.e., in a three-dimensional set) whereas $K$ is kept constant.

We propose to call $\tilde{V}^{i}$ the collective indirect utility of agent $i$, to reflect the fact that the definition of $\tilde{V}^{i}$ implicitly includes the sharing function $\rho_{i}$ and hence an outcome of the collective decision process. In particular, in contrast to the individual indirect utility $V^{i}$, the collective indirect utility $\tilde{V}^{i}$ can be defined only in reference to a particular decision process. Note that whenever normative judgments are at stake, the collective indirect utility is the relevant concept, since it measures the level of utility that will ultimately be reached by each agent, taking into account the redistribution that will take place within the household.

Determination of public expenditures.-We can now characterize the efficiency conditions for public-good expenditures. They take the standard Bowen-Lindahl-Samuelson form. Namely, with an interior solution, the first-order conditions for problem (2) give

$$
\frac{\partial U^{1} / \partial K}{\partial U^{1} / \partial C}+\frac{\partial U^{2} / \partial K}{\partial U^{2} / \partial C}=1 .
$$

Equivalently, one can use the two-stage representation and express the conditions in terms of individual indirect utilities. The optimal choice of $\left(\rho_{1}, \rho_{2}, K\right)$ solves

$$
\begin{aligned}
& \max _{\rho_{1}, \rho_{2}, K} \lambda V^{1}\left(w_{1}, \rho_{1}, K\right)+(1-\lambda) V^{2}\left(w_{2}, \rho_{2}, K\right) \\
& \text { subject to } \rho_{1}+\rho_{2}+K=Y,
\end{aligned}
$$


which gives

$$
\lambda \frac{\partial V^{1}}{\partial \rho}=(1-\lambda) \frac{\partial V^{2}}{\partial \rho}=\lambda \frac{\partial V^{1}}{\partial K}+(1-\lambda) \frac{\partial V^{2}}{\partial K}
$$

hence

$$
\frac{\partial V^{1} / \partial K}{\partial V^{1} / \partial \rho}+\frac{\partial V^{2} / \partial K}{\partial V^{2} / \partial \rho}=1
$$

The ratio $\left(\partial V^{i} / \partial K\right) /\left(\partial V^{i} / \partial \rho\right)$ is $i$ s marginal willingness to pay for the public good; condition (7) states that the individual's marginal willingness to pay must add up to the price of the public good.

Finally, the same condition can be expressed in terms of collective indirect utilities. After simple calculations, one gets

$$
\alpha \frac{\partial \tilde{V}^{1} / \partial K}{\partial \tilde{V}^{1} / \partial Y}+(1-\alpha) \frac{\partial \tilde{V}^{2} / \partial K}{\partial \tilde{V}^{2} / \partial Y}=1,
$$

where

$$
\alpha=\frac{\partial \rho_{1}}{\partial Y}+\frac{\partial \rho_{1}}{\partial z} \frac{\partial \zeta}{\partial Y} .
$$

In words, if nonlabor income is changed by one dollar, $z$ being adjusted so as to keep $K$ constant, $\alpha$ is the fraction of this change borne by member 1 (obviously, $1-\alpha$ is borne by member 2). Again, (8) expresses that agents are indifferent to one marginal dollar being spent on the public good, the cost being divided between the agents according to the proportions thus defined.

Separability.-For general preferences the level of public consumption influences the optimal choice of consumption and labor supply through two channels: an income effect (i.e., more public expenditures means less total private consumption and hence [presumably] a tighter private budget for both members) and the direct impact of public expenditures on the consumption-leisure trade-off. In the separable case (S), however, the second effect disappears. Technically, the second-stage problem (4) becomes

$$
\begin{aligned}
& \max _{L^{i}, C^{i}} u^{i}\left(L^{i}, C^{i}\right) \\
& \text { subject to } w_{i} L^{i}+C^{i}=w_{i}+\rho_{i} .
\end{aligned}
$$

These separability properties at the individual level are preserved in 
the optimal value function of the household, although in a somewhat specific way. ${ }^{11}$

One can also express separability in terms of indirect utilities. The individual indirect utility $V^{i}$ is such that the individual wage and sharing rule are separable from the public expenditure $K$. Specifically, let $v^{i}\left(w_{i}, \rho_{i}\right)$ denote the value of program (9); note that $v^{i}$ does not depend on $K$ directly. Then the individual's indirect utility function $V^{i}$ is defined by

$$
V^{i}\left(w_{i}, \rho_{i}, K\right)=W^{i}\left[v^{i}\left(w_{i}, \rho_{i}\right), K\right] .
$$

More interesting, the definition of collective indirect utility $\tilde{V}^{i}$ no longer requires the presence of a distribution factor. To see why, assume away for a moment the distribution factor (so that all functions depend only on $\left.\left(w_{1}, w_{2}, Y\right)\right)$, and let $y$ denote the portion of nonlabor income not devoted to the purchase of the public good:

$$
y=Y-K\left(w_{1}, w_{2}, Y\right) .
$$

Clearly, $y$ can be positive or negative. Again, consider some open subset $\mathcal{O}^{\prime}$ of $\mathcal{K}$ such that $\partial K / \partial Y \neq 1$ on $\mathcal{O}^{\prime}$; that is, an additional dollar of nonlabor income would not be entirely spent on the public good, a natural requirement. By the implicit function theorem, the equation above allows one to express $Y$ as some function $\Upsilon$ of $\left(w_{1}, w_{2}, y\right)$. Then we can define, over $\mathcal{O}^{\prime}$, the function $\tilde{V}^{i}$ by

$$
\tilde{V}^{i}\left(w_{1}, w_{2}, y\right)=V^{i}\left[w_{i}, \rho_{i}\left(w_{1}, w_{2}, \Upsilon\left(w_{1}, w_{2}, y\right)\right)\right] .
$$

Intuitively, since the subutility $u^{i}$ can be defined independently of $K$, the additional dimension provided by the distribution factor is no longer needed.

\section{Collective Analysis of Welfare: An Illustration}

This subsection illustrates the way collective models allow one to study "targeting" and, more generally, issues related to intrahousehold dis-

${ }^{11}$ If individual utilities satisfy the separability property (S), then from eq. (3) above, the household's MRS between individual $i$ 's leisure and total private consumption $C$ satisfies the equation

$$
\frac{\partial H / \partial L^{i}}{\partial H / \partial C}=\frac{\partial u^{i} / \partial L^{i}}{\partial u^{i} / \partial C}
$$

where the right-hand side depends only on $L^{i}$ and $C^{i}$. This means that if we control for individual consumptions, the household MRS does not depend on public-good expenditures $K$. The property must, however, be handled with care, since it does not lead to the standard separability tests of consumer theory. The right-hand-side expression in the equation above is the MRS of individual $i$, which is taken at $\left(L^{i}, C^{i}\right)$. Since $C^{i}$ is not observed, one cannot directly test this property in the usual way. We are indebted to the editor, Fernando Alvarez, for pointing this out to us. 
tribution of power and its impact on behavior. Our claim is that an explicit formalization of individual preferences over private and public consumption is crucial for the purposes of analyzing the welfare implications of policy reforms and for understanding issues such as child poverty. The implications are far-reaching, and they are relevant for policy both in the context of developing countries and for industrialized ones.

The question we consider here is the following: How does a change in the distribution of power within the household-that is, here, a change in the Pareto weight $\lambda$-affect the expenditure on the public good? In particular, when is it the case that an improvement of the mother's position (say, because a benefit is now targeted to her) increases expenditures on children? We shall see that an answer can readily be given in the theoretical context just developed.

For notational simplicity, we define $\rho \equiv \rho_{1}$; then $\rho_{2}=Y-K-\rho=$ $y-\rho$. Condition (7) above can be written as

$$
\lambda \frac{\partial V^{1}\left(w_{1}, \rho, K\right)}{\partial \rho_{1}}=(1-\lambda) \frac{\partial V^{2}\left(w_{2}, Y-\rho-K, K\right)}{\partial \rho_{2}}
$$

and

$$
\operatorname{MWP}^{1}\left(w_{1}, \rho, K\right)+\operatorname{MWP}^{2}\left(w_{2}, Y-\rho-K, K\right)=1,
$$

where $\mathrm{MWP}^{i}$ denotes $i$ 's marginal willingness to pay for the public good. Using the implicit function theorem on these first-order conditions, we get

$$
\begin{aligned}
& \frac{\partial \rho}{\partial \lambda}=\frac{1}{D}\left(\frac{\partial \mathrm{MWP}^{1}}{\partial K}+\frac{\partial \mathrm{MWP}^{2}}{\partial K}-\frac{\partial \mathrm{MWP}^{2}}{\partial \rho_{2}}\right)\left(\frac{\partial V^{1}}{\partial \rho_{1}}+\frac{\partial V^{2}}{\partial \rho_{2}}\right), \\
& \frac{\partial K}{\partial \lambda}=-\frac{1}{D}\left(\frac{\partial \mathrm{MWP}^{1}}{\partial \rho_{1}}-\frac{\partial \mathrm{MWP}}{\partial \rho_{2}}\right)\left(\frac{\partial V^{1}}{\partial \rho_{1}}+\frac{\partial V^{2}}{\partial \rho_{2}}\right)
\end{aligned}
$$

where $D$ is given by

$$
\begin{aligned}
D= & \left\{\lambda \frac{\partial^{2} V^{1}}{\partial \rho_{1} \partial K}-(1-\lambda)\left[\frac{\partial^{2} V^{2}}{\partial \rho_{2} \partial K}-\frac{\partial^{2} V^{2}}{\left(\partial \rho_{2}\right)^{2}}\right]\right\}\left(\frac{\partial \mathrm{MWP}^{1}}{\partial \rho_{1}}-\frac{\partial \mathrm{MWP}^{2}}{\partial \rho_{2}}\right) \\
& -\left(\frac{\partial \mathrm{MWP}}{\partial K}+\frac{\partial \mathrm{MWP}}{\partial K}-\frac{\partial \mathrm{MWP}^{2}}{\partial \rho_{2}}\right)\left[\lambda \frac{\partial^{2} V^{1}}{\left(\partial \rho_{1}\right)^{2}}+(1-\lambda) \frac{\partial^{2} V^{2}}{\left(\partial \rho_{2}\right)^{2}}\right] .
\end{aligned}
$$

We assume that preferences are such that the "goods" $\rho$ and $K$ are normal; that is, an increase in nonlabor income boosts both private and public consumption. Then $\mathrm{MWP}^{i}$ is increasing in $\rho_{i}$ and decreasing in $K$, and the first expression in parentheses in $\partial \rho / \partial \lambda$ is negative. Also, 
$\partial V^{1} / \partial \rho_{1}$ and $\partial V^{2} / \partial \rho_{2}$ are both positive. Finally, we may, without loss of generality, assume that the difference

$$
D \mathrm{MWP} \equiv \frac{\partial \mathrm{MWP}^{1}}{\partial \rho_{1}}-\frac{\partial \mathrm{MWP}^{2}}{\partial \rho_{2}}
$$

is positive. Then $\partial K / \partial \lambda$ has the same sign as $\partial \rho / \partial \lambda$. Increasing $\lambda$ can thus either increase $K$ and $\rho$ or decrease both. But the second case is impossible, because an increase in 1's weight would then reduce the utility of 1 , a contradiction. We conclude that $\partial K / \partial \lambda \geq 0$ and $\partial \rho / \partial \lambda \geq$ 0 .

We can summarize our findings in the following proposition.

Proposition 1. Assume that preferences are such that each member $i$ 's marginal willingness to pay for the public good is decreasing in the level of the public good and increasing in the member's share $\rho_{i}$. Then a marginal change in a member's Pareto weight increases the household's expenditures on the public good if and only if the marginal willingness to pay of this member is more sensitive to changes in his or her share than that of the other member.

For instance, if individual preferences are Cobb-Douglas,

$$
U^{i}\left(c^{i}, L^{i}, K\right)=\alpha_{i} \log L^{i}+\left(1-\alpha_{i}\right) \log C^{i}+\delta_{i} \log K,
$$

and if we assume that the Pareto weight of member $1, \lambda=l w_{1} /\left(l w_{1}+\right.$ $w_{2}$ ), is increasing in some parameter $l$, straightforward computations show that the partial $\partial K / \partial l$ is positive if and only if $\delta_{1}>\delta_{2}$ (which is indeed equivalent to $\left.\partial \mathrm{MWP}^{1} / \partial \rho>\partial \mathrm{MWP}^{2} / \partial \rho\right)$; note, however, that even with $\delta_{1}>\delta_{2}$, it may be the case that

$$
\mathrm{MWP}^{1}=\delta_{1} \frac{w_{1}+\rho_{1}}{K}<\mathrm{MWP}^{2}=\delta_{2} \frac{w_{2}+\rho_{2}}{K}
$$

(particularly if $w_{2}$ is large with respect to $w_{1}$ ).

\section{Identifiability}

The identifiability question relates to our ability to recover individual preferences and the Pareto weight from the sole observation of labor supplies and the expenditures on the public good, as functions of wages and nonlabor income. As argued in Section I, identifiability is a key requirement for guaranteeing the empirical relevance of the normative approach described above: Despite all the conceptual insights it helps formalize, the collective approach would be of little help if the concepts at stake could not be recovered from observed behavior, because the analysis would then have limited empirical content.

Technically, the setting is fully determined by the 3-tuple $\left(U^{1}, U^{2}\right.$, 
$\lambda)$, where $U^{i}$ and $\underset{\tilde{U}}{\lambda}$ are functions mapping $\mathbb{R}^{3}$ to $\mathbb{R}$. Two 3-tuples $\left(U^{1}\right.$, $\left.U^{2}, \lambda\right)$ and $\left(\tilde{U}^{1}, \tilde{U}^{2}, \tilde{\lambda}\right)$ are equivalent if (i) $U^{i}=f^{i}\left(\tilde{U}^{i}\right)$ for some increasing $f^{i}$ and (ii) for all $\left(w_{1}, w_{2}, Y, z\right)$ in $\mathcal{K}$, the solution to the household problem (2) is the same for $\left(U^{1}, U^{2}, \lambda\right)$ and $\left(\tilde{U}^{1}, \tilde{U}^{2}, \tilde{\lambda}\right)$. Condition $\mathrm{i}$ implies that the Pareto sets for $\left(U^{1}, U^{2}\right)$ and $\left(\tilde{U}^{1}, \tilde{U}^{2}\right)$ are always identical, whereas condition ii imposes in addition that the location of the decision on this frontier is always the same for the two 3-tuples. We define a structure as a set of equivalent 3-tuples.

To any given structure, one can associate labor supply and children expenditure functions $L^{1}\left(w_{1}, w_{2}, Y, z\right), L^{2}\left(w_{1}, w_{2}, Y, z\right)$, and $K\left(w_{1}, w_{2}\right.$, $Y, z)$, defined as the solution to the household problem (2). The structure is identifiable if this mapping from structures to behavior functions is one to one, that is, if two different structures cannot generate the same labor supply and children expenditure functions. Equivalently, the structure is identifiable if (at most) one structure corresponds to any given labor supply and children expenditure functions.

\section{A. Identifiability: Separability and Distribution Factors}

In general, preferences and the Pareto weights are not identifiable: An observed reduced form, which relates each person's labor supply and expenditures on children to wages $\left(w_{1}\right.$ and $\left.w_{2}\right)$ and nonlabor income $(Y)$, can be generated by a continuum of different structural models. However, we show below that under two separate conditions, identifiability obtains in the sense that if a structural model is compatible with the "reduced-form" functions, all of which are observable, then this structural model is unique.

Most straightforwardly, if we know of a distribution factor, namely, a variable affecting the Pareto weights but not preferences of either individual, then we show below that at most one structural model corresponds to the observed reduced form. ${ }^{12}$ If no distribution factor is available, then we show that the uniqueness result is preserved, but only within the class of separable utility functions. This means that if a reduced form is compatible with separable preferences, those preferences will be unique. However, there will be a continuum of nonseparable preferences generating the same reduced form.

It turns out that separability has implications for the reduced-form conditional labor supply functions. These implications can provide the basis for a statistical test in an empirical model. If these conditions are not valid, preferences are not separable and thus are identifiable only on the basis of a distribution factor. If they are valid, we can

\footnotetext{
${ }^{12}$ Lack of a solution would imply that the reduced forms do not correspond to the solution of a collective optimization problem.
} 
identify a separable preference structure that generates the reduced form. Nevertheless the true preferences might still be nonseparable, but there is no information to establish this unless there is a distribution factor.

\section{B. Identifiability with Distribution Factors}

We now proceed to show that the knowledge of $L^{1}, L^{2}$, and $K$ (as functions of $w_{1}, w_{2}, Y$, and $z$ ) is sufficient for identifiability of the underlying structure. The general strategy goes as follows: We first consider the information embodied in the labor supply function. We show that the basic intuition of the private consumption case can readily be extended; that is, it is still possible to identify individual consumptions up to an additive constant (which may depend on the level of public-good expenditures). This, in turn, allows us to recover individual indirect utilities up to an increasing function of the public good. We then show that by using the public expenditure function, we are able to identify the structure.

Labor supply and the sharing rule.-We first concentrate on private expenditures and fix public expenditures to some arbitrary level $\bar{K}$. Thus technically we consider as above some open subset $\mathcal{O}$ of $\mathcal{K}$ such that $\partial K^{*} / \partial z$ does not vanish on $\mathcal{O}$, and we impose the condition $K^{*}\left(w_{1}\right.$, $\left.w_{2}, Y, z\right)=\bar{K}$, which by the implicit function theorem is equivalent to $z=\zeta\left(w_{1}, w_{2}, Y, \bar{K}\right)$. As above, we use the notation $\rho \equiv \rho_{1}$ and $\rho_{2}=$ $Y-K-\rho=y-\rho$. Then $L^{1}, L^{2}$, and $\rho$ are functions of $\left(w_{1}, w_{2}, Y\right.$, $\left.\zeta\left(w_{1}, w_{2}, Y, \bar{K}\right)\right)$ and hence of $\left(w_{1}, w_{2}, Y\right)$ since $\bar{K}$ is fixed. Using for now the change in variable $y=Y-\bar{K}$, we can express $L^{1}, L^{2}$, and $\rho$ as functions of $\left(w_{1}, w_{2}, y\right)$; for notational simplicity, we still denote these functions $L^{1}, L^{2}$, and $\rho$, since no confusion is to be feared.

Now, consider the two programs in (4):

$$
\begin{aligned}
& \max _{L^{1}, C^{1}} U^{1}\left(L^{1}, C^{1}, \bar{K}\right) \\
& \text { subject to } w_{1} L^{1}+C^{1}=w_{1}+\rho
\end{aligned}
$$

and

$$
\begin{aligned}
& \max _{L^{2}, C^{2}} U^{2}\left(L^{2}, C^{2}, \bar{K}\right) \\
& \text { subject to } w_{2} L^{2}+C^{2}=w_{2}+y-\rho .
\end{aligned}
$$

From a theorem in Chiappori (1992), the knowledge of the two labor supply functions allows one to recover the sharing rule and the individual utilities up to an increasing constant; moreover, the constant is 
welfare irrelevant, in the sense that it does not affect the indirect utility. A formal statement follows.

Lemma 1. For any given $\bar{K}$, assume that two 3-tuples $\left(U^{1}, U^{2}, \rho\right)$ and $\left(\hat{U}^{1}, \hat{U}^{2}, \hat{\rho}\right)$ generate for all $\left(w_{1}, w_{2}, y\right)$ the same labor supplies in programs (13) and (14). Then generically on the 3-tuple $\left(U^{1}, U^{2}, \rho\right)$, there exists a constant $A(\bar{K})$ such that, for all $\left(w_{1}, w_{2}, y\right)$,

$$
\begin{aligned}
\hat{\rho}\left(w_{1}, w_{2}, y\right) & =\rho\left(w_{1}, w_{2}, y\right)+A(\bar{K}), \\
\hat{U}^{1}\left(L^{1}, C^{1}, \bar{K}\right) & =f^{1}\left[U^{1}\left(L^{1}, C^{1}-A(\bar{K}), \bar{K}\right), \bar{K}\right], \\
\hat{U}^{2}\left(L^{2}, C^{2}, \bar{K}\right) & =f^{2}\left[U^{2}\left(L^{2}, C^{2}+A(\bar{K}), \bar{K}\right), \bar{K}\right],
\end{aligned}
$$

where $f^{1}$ and $f^{2}$ are twice continuously differentiable mappings, increasing in their first argument. Moreover, the individual indirect utilities are such that, for all $\left(w_{1}, w_{2}, y\right)$ (with obvious notation),

$$
\begin{aligned}
\hat{V}^{1}\left(w_{1}, \hat{\rho}, \bar{K}\right) & =f^{1}\left[V^{1}\left(w_{1}, \rho, \bar{K}\right), \bar{K}\right], \\
\hat{V}^{2}\left(w_{2}, y-\hat{\rho}, \bar{K}\right) & =f^{2}\left[V^{2}\left(w_{2}, y-\rho, \bar{K}\right), \bar{K}\right] .
\end{aligned}
$$

In particular, the collective indirect utilities corresponding to the two solutions coincide, again up to an increasing function of $\bar{K}$.

Proof. See proposition 4 in Chiappori (1992). The result is "generic" in the sense that it requires a "regularity" assumption on labor supplies (condition R in Chiappori's article); specifically, the set of labor supply functions for which the result does not hold is characterized by a partial differential equation (PDE). The only adjustment with respect to Chiappori's model is that in our context, both the additive constant and the increasing mappings $f^{i}$ are indexed by the level of public expenditures. That is, the indirect utilities are such that, for any $\left(w_{1}, w_{2}, R, K\right)$,

$$
\begin{aligned}
& \hat{V}^{1}\left(w_{1}, R, K\right)=f^{1}\left[V^{1}\left(w_{1}, R-A(K), K\right), K\right], \\
& \hat{V}^{2}\left(w_{2}, R, K\right)=f^{2}\left[V^{2}\left(w_{2}, R+A(K), K\right), K\right] .
\end{aligned}
$$

Thus the functions $\hat{V}^{i}$ and $V^{i}$ are different; but the value taken by $\hat{V}^{1}$ $\left(\hat{V}^{2}\right)$ for $R=\hat{\rho}(R=y-\hat{\rho})$ and the value taken by $V^{1}\left(V^{2}\right)$ for $R=$ $\rho(R=y-\rho)$ coincide up to an increasing function of $K$. QED

Lemma 1 states that individual preferences and the sharing rule are identifiable up to some additive constant, which may clearly depend on $\bar{K}$. However, this constant does not affect the value taken by the individual indirect utilities $V^{i}$ and their derivatives with respect to private shares 
$\rho_{i \cdot}{ }^{13}$ As always, (indirect) utilities can be identified only up to an increasing monotonic transformation; again, this transformation may depend on $\bar{K}$. In other words, one can identify a 3-tuple $\left(V^{1}, V^{2}, \rho\right)$ such that any other solution $\left(\hat{V}^{1}, \hat{V}^{2}, \hat{\rho}\right)$ must satisfy condition $(15)$. From now on, thus, $\left(V^{1}, V^{2}, \rho\right)$ will be known functions; what remain to be identified are the functions $f^{i}$. Finally, note that additional, overidentifiability restrictions are generated.

Preferences for public consumption.-We now consider the demand for public goods. Any solution $\left(\hat{V}^{1}, \hat{V}^{2}, \hat{\rho}\right)$, under the assumption of an interior solution, must satisfy the following first-order conditions:

$$
\frac{\partial \hat{V}^{1} / \partial K}{\partial \hat{V}^{1} / \partial \rho_{1}}+\frac{\partial \hat{V}^{2} / \partial K}{\partial \hat{V}^{2} / \partial \rho_{2}}=1
$$

From (15) one can compute the partials of $\hat{V}^{i}$; hence

$$
\begin{gathered}
\frac{1}{\partial V^{1} / \partial \rho_{1}} \frac{\partial f^{1} / \partial K}{\partial f^{1} / \partial V}+\frac{1}{\partial V^{2} / \partial \rho_{2}} \frac{\partial f^{2} / \partial K}{\partial f^{2} / \partial V}= \\
1-\left(\frac{\partial V^{1} / \partial K}{\partial V^{1} / \partial \rho_{1}}+\frac{\partial V^{2} / \partial K}{\partial V^{2} / \partial \rho_{1}}\right)
\end{gathered}
$$

where the $V^{i}$ are known and the $f^{i}$ are unknown. Clearly, only the ratio $\left(\partial f^{i} / \partial K\right) /\left(\partial f^{i} / \partial V\right)$ can (at best) be identifiable, reflecting the fact that $f^{i}$ is (at best) identifiable up to some increasing transform only. Hence if $\Phi$ is defined by $\phi^{i}\left(V^{i}, K\right)=\left(\partial f^{i} / \partial K\right) /\left(\partial f^{i} / \partial V\right)$, then (16) can be rewritten as

$$
\begin{gathered}
\frac{1}{\partial V^{1} / \partial \rho_{1}} \phi^{1}\left(V^{1}, K\right)+\frac{1}{\partial V^{2} / \partial \rho_{2}} \phi^{2}\left(V^{2}, K\right)= \\
1-\left(\frac{\partial V^{1} / \partial K}{\partial V^{1} / \partial \rho_{1}}+\frac{\partial V^{2} / \partial K}{\partial V^{2} / \partial \rho_{1}}\right) .
\end{gathered}
$$

We now proceed to show that generically (in a sense that will be made precise later), the solution to this equation (if any) is unique. The result comes from the fact that the unknowns are functions of only two variables, whereas the equation depends in general on four variables $\left(w_{1}\right.$, $w_{2}, Y$, and $z$ ). To use this feature, let us first note that (17) is linear in $\phi^{1}$ and $\phi^{2}$. Thus, if there exist two distinct solutions $\left(\phi^{1}, \phi^{2}\right)$ and $\left(\phi^{\prime 1}\right.$,

\footnotetext{
${ }^{13}$ The functions $V^{1}$ and $V^{2}$ are identified only up to the same additive constant as the sharing rule. However, the value taken by the functions (and their derivatives) at this sharing rule is the same for all solutions.
} 
$\left.\phi^{\prime 2}\right)$, the differences $\psi^{i}=\phi^{i}-\phi^{i i}$ must satisfy the homogeneous equation

$$
\frac{1}{\partial V^{1} / \partial \rho_{1}} \psi^{1}\left(V^{1}, K\right)+\frac{1}{\partial V^{2} / \partial \rho_{2}} \psi^{2}\left(V^{2}, K\right)=0 .
$$

At any point such that $\psi^{i}\left(V^{i}, K\right) \neq 0$, one must have that $\psi^{j}\left(V^{j}\right.$, $K) \neq 0$ for $i \neq j$, and (18) can be written as

$$
\log \psi^{1}\left(V^{1}, K\right)-\log \psi^{2}\left(V^{2}, K\right)=\log \left(\frac{\partial V^{1} / \partial \rho_{1}}{\partial V^{2} / \partial \rho_{2}}\right),
$$

which requires that the right-hand-side function

$$
\log \left(\frac{\partial V^{1} / \partial \rho_{1}}{\partial V^{2} / \partial \rho_{2}}\right)
$$

be the sum of a function of $\left(V^{1}, K\right)$ and a function of $\left(V^{2}, K\right)$. For generic functions $V^{1}, V^{2}$, and $\rho$, this property is almost never satisfied; hence it must be the case that

$$
\psi^{1}\left(V^{1}, K\right)=\psi^{2}\left(V^{2}, K\right)=0
$$

almost everywhere. A more precise statement can be found in the Appendix. We conclude that, when equation (17) has a solution, the solution is generically unique.

Lemma 1 states that the labor supply functions allow one to identify the collective indirect utilities up to an increasing function of $K$. We have shown here that once expenditures on children are taken into account, identifiability obtains up to an increasing transform; that is, the corresponding indirect preferences are exactly identified.

It remains to see whether a solution to equation (17) exists at all; this generates additional overidentifiability restrictions, an example of which is provided below for the Cobb-Douglas example. Finally, once a particular cardinalization has been chosen, one can recover the Pareto weight from the first-order conditions:

$$
\lambda \frac{\partial V^{1}}{\partial \rho}=(1-\lambda) \frac{\partial V^{2}}{\partial \rho} \Leftrightarrow \lambda=\frac{\partial V^{2} / \partial \rho}{\left(\partial V^{1} / \partial \rho\right)+\left(\partial V^{2} / \partial \rho\right)} .
$$

Our results can be summarized in the following statement.

Proposition 2. Let $L^{1}, L^{2}$, and $K$ be given functions of $w_{1}, w_{2}, Y$, and $z$. Generically, the knowledge of these functions identifies the corresponding collective indirect utilities up to some increasing mappings. Moreover, for any particular cardinalization, the Pareto weight is exactly identified. 


\section{Implications of Separability for the Reduced-Form Labor Supply}

Functions in the Absence of Distribution Factors

To provide the basis for a test of separability of leisure and consumption from expenditure on children based on observable quantities, we derive the implications of this restriction on the reduced form starting from the equilibrium relationship

$$
\frac{\partial H / \partial L^{i}}{\partial H / \partial C}=\frac{\partial u^{i} / \partial L^{i}}{\partial u^{i} / \partial C}
$$

which depends only on $\left(L^{i}, C^{i}\right)$ and not on $K$. However, taking this property to data is a delicate task because $C^{i}$ is not observed.

In what follows, we assume that consumption is always a normal good at the individual level and that aggregate private consumption $C$ is an increasing function of nonlabor income. We first define $\tilde{L}^{i}$ as $i$ 's conditional demand for leisure (i.e., $i$ 's demand for leisure as a function of $w_{1}, w_{2}$, and $\left.C\right) ; ;^{14}$ note that the $\tilde{L}^{i}$ are known from the data.

Since consumption is a normal good, the individual first-order conditions can be inverted and expressed as

$$
C^{i}=\phi^{i}\left(w_{i}, L^{i}\right)
$$

where $\phi^{i}$ is increasing. It follows that the conditional demands for leisure $\tilde{L}^{i}$ must satisfy a relationship of the form

$$
\phi^{1}\left(w_{1}, \tilde{L}^{1}\left(w_{1}, w_{2}, C\right)\right)+\phi^{2}\left(w_{2}, \tilde{L}^{2}\left(w_{1}, w_{2}, C\right)\right)-C=0
$$

for some well-chosen $\phi^{i}$. In a lemma presented in the Appendix, we show that, generically, this is not the case; that is, such $\phi^{i}$ do not exist unless the functions $\tilde{L}^{i}$ satisfy necessary conditions, which take the form of PDEs. These can be hard to take to the data, but their existence does show that separability has implications for the observable relationships, even in the absence of a distribution factor. Moreover, the conditions

${ }^{14}$ Technically, let $C\left(w_{1}, w_{2}, y\right)$ denote the aggregate consumption function. From the implicit function theorem, the relationship $C=C\left(w_{1}, w_{2}, y\right)$ can be inverted into $y=$ $\Gamma\left(w_{1}, w_{2}, C\right)$. Plugging this into the individual demands for leisure gives $\tilde{L}^{i}\left(w_{1}, w_{2}, C\right)=$ $L^{i}\left(w_{1}, w_{2}, \Gamma\left(w_{1}, w_{2}, C\right)\right)$. 
can readily be tested on a parametric form. Assume, for instance, that conditional labor supply has the quadratic form

$$
\begin{aligned}
\tilde{L}^{1}\left(w_{1}, w_{2}, C\right)= & a_{1}^{1} w_{1}+a_{2}^{1} w_{2}+b_{1}^{1}\left(w_{1}\right)^{2}+b_{2}^{1}\left(w_{2}\right)^{2}+c^{1} w_{1} w_{2}+d^{1} C \\
& +e^{1} C^{2}+f^{1} \\
\tilde{L}^{2}\left(w_{1}, w_{2}, C\right)= & a_{1}^{2} w_{1}+a_{2}^{2} w_{2}+b_{1}^{2}\left(w_{1}\right)^{2}+b_{2}^{2}\left(w_{2}\right)^{2}+c^{2} w_{1} w_{2}+d^{2} C \\
& +e^{2} C^{2}+f^{2} .
\end{aligned}
$$

For generic values of the parameters, it can be checked whether the PDEs are not satisfied, that is, that one cannot find two functions $\phi^{1}$ and $\phi^{2}$ such that (20) is satisfied. In addition, one can derive sufficient conditions for the existence of $\phi^{1}$ and $\phi^{2}$. For instance, the conditions are satisfied if $e^{1}=e^{2}=0 .{ }^{15}$

The key point of this result is that it provides the basis for testing for separability, within the context of an empirical analysis, when a distribution factor is unavailable; we exploit both the variation implied by unearned income and the particular structure implied by separability. The conclusion may be that the reduced form is not compatible with any separable preference structure. In the presence of a distribution factor, a simpler test can be devised.

\section{Identification under Separability}

The proof of identifiability in the presence of distribution factors applies here; note, however, that we are now solving for separable preferences. The knowledge of labor supplies and expenditures on children $K$ as functions of wages and nonlabor income is sufficient only to recover the underlying structure. Indeed, the only role of the distribution factor was to introduce an additional, observable, dimension that allows wages and nonlabor incomes to vary whereas expenditures on children are kept constant. This was needed because changes in expenditures on children would generally modify the individual trade-off between leisure and private consumption, hence hampering the analysis of labor supply. Clearly, this concern does not exist in the separable case. In addition, it is possible that no distribution factor is available, in the sense that any such variable may be considered as affecting preferences. In this case, separability is a sufficient condition that allows identifiability.

\footnotetext{
${ }^{15}$ The computations are particularly tedious and are not reported here. They are available from the authors.
} 
Programs (13) and (14) become

$$
\begin{aligned}
& \max _{L^{1}, C^{1}} u^{1}\left(L^{1}, C^{1}\right) \\
& \text { subject to } w_{1} L^{1}+C^{1}=w_{1}+\rho
\end{aligned}
$$

and

$$
\begin{aligned}
& \max _{L^{2}, C^{2}} u^{2}\left(L^{2}, C^{2}\right) \\
& \text { subject to } w_{2} L^{2}+C^{2}=w_{2}+y-\rho .
\end{aligned}
$$

While variations in wages and nonlabor income do change expenditures on children, this effect is irrelevant for the study of labor supply since the only impact occurs through income effects, which are captured by the sharing rule anyway. In practice, labor supplies can be estimated as functions of wages and residual nonlabor income $y=Y-K$; changes in $K$ are fully captured through their impact on $y$. Then the proof proceeds as before. Namely, programs (21) and (22) identify the value of the individual utilities and of their derivatives; then the first-order conditions for public expenditures generically recover the utility functions $W^{1}$ and $W^{2}$, the argument being exactly the same as above.

\section{E. A General Example}

We now consider an example that illustrates the limits of identification in the absence of a distribution factor. Consider the following nonseparable preferences:

$$
\begin{aligned}
& U^{1}\left(L^{1}, C^{1}, K\right)=\alpha_{1} \log \left(L^{1}+\gamma K\right)+\left(1-\alpha_{1}\right) \log C^{1}+\delta_{1} \log K, \\
& U^{2}\left(L^{2}, C^{2}, K\right)=\alpha_{2} \log L^{2}+\left(1-\alpha_{2}\right) \log C^{2}+\delta_{2} \log K .
\end{aligned}
$$

Note that $U^{1}$ is not separable in the sense of (S) unless the coefficient $\gamma$ is zero. Assume, as above, that member 1's Pareto weight $\lambda$ is proportional to wages, that is,

$$
\lambda=\frac{l w_{1}}{l w_{1}+w_{2}} .
$$

Straightforward calculations give

$$
\begin{gathered}
L^{1}=\left[\frac{\alpha_{1} l\left(1-\gamma w_{1}\right)-\gamma\left(\delta_{1} l w_{1}+\delta_{2} w_{2}\right)}{1-\gamma w_{1}}\right] \frac{w_{1}+w_{2}+y}{\left(1+\delta_{1}\right) l w_{1}+\left(1+\delta_{2}\right) w_{2}}, \\
L^{2}=\frac{\alpha_{2}}{\left(1+\delta_{1}\right) l w_{1}+\left(1+\delta_{2}\right) w_{2}}\left(w_{1}+w_{2}+y\right),
\end{gathered}
$$




$$
\begin{aligned}
& K=\frac{\delta_{1} l w_{1}+\delta_{2} w_{2}}{\left[\left(1+\delta_{1}\right) l w_{1}+\left(1+\delta_{2}\right) w_{2}\right]\left(1-\gamma w_{1}\right)}\left(w_{1}+w_{2}+y\right), \\
& C=C^{1}+C^{2}=\frac{\left(1-\alpha_{1}\right) l w_{1}+\left(1-\alpha_{2}\right) w_{2}}{\left(1+\delta_{1}\right) l w_{1}+\left(1+\delta_{2}\right) w_{2}}\left(w_{1}+w_{2}+y\right),
\end{aligned}
$$

and conditional demands for leisure are

$$
\begin{aligned}
\tilde{L}^{1} & =\left[\frac{\alpha_{1} l\left(1-\gamma w_{1}\right)-\gamma\left(\delta_{1} l w_{1}+\delta_{2} w_{2}\right)}{1-\gamma w_{1}}\right] \frac{C}{\left(1-\alpha_{1}\right) l w_{1}+\left(1-\alpha_{2}\right) w_{2}}, \\
\tilde{L}^{2} & =\frac{\alpha_{2}}{\left(1-\alpha_{1}\right) l w_{1}+\left(1-\alpha_{2}\right) w_{2}} C .
\end{aligned}
$$

Not surprisingly, the conditions characterizing separability are satisfied when $\gamma=0$ (i.e., when the initial utilities are indeed separable). More surprisingly, however, the conditions are also satisfied when $\gamma \neq 0$. Indeed, property (20) is satisfied when $\phi^{1}$ and $\phi^{2}$ are defined by

$$
\begin{aligned}
& \phi^{1}\left(w_{1}, L^{1}\right)=-\frac{1-\alpha_{1}}{\gamma\left(\alpha_{1}+\delta\right)}\left(1-\gamma w_{1}\right) L^{1}, \\
& \phi^{2}\left(w_{2}, L^{2}\right)=\left[\left(\frac{1-\alpha_{2}}{\alpha_{2}}-\frac{1-\alpha_{1}}{\alpha_{1}+\delta_{1}} \frac{\delta_{2}}{\alpha_{2}}\right) w_{2}+\frac{1-\alpha_{1}}{\gamma\left(\alpha_{1}+\delta_{1}\right)} \frac{\alpha_{1} l}{\alpha_{2}}\right] L^{2} .
\end{aligned}
$$

While this result may seem paradoxical, it is in fact fully compatible with the previous results, and it helps one to understand their exact scope. In the absence of a distribution factor, the model is not identifiable; hence the reduced forms (26) are consistent with the structural model defined by (23) and (24) but also with a continuum of different structural models. As it turns out, this particular example is such that one of these alternative structures involves separable preferences. In other words, there exist two separable utilities $\hat{U}^{1}$ and $\hat{U}^{2}$, obviously different from the $U^{1}$ and $U^{2}$ defined by (23), that generate the same observable labor supply functions. ${ }^{16}$ This stresses a point made earlier. If the reduced forms have been generated by some separable structure, they must satisfy conditions (8) above. But the converse is not true. If the reduced forms satisfy conditions (20), they are consistent with separable preferences, but this does not exclude the possibility that they have been generated by some nonseparable structure. Separability is an

\footnotetext{
${ }^{16}$ This case is peculiar in the sense that, in general, arbitrary reduced forms are not consistent with any separable structure, as illustrated by the derivation of the implications of separability provided above.
} 
identifying assumption, precisely in the sense that (without distribution factors) uniqueness obtains only within the class of separable structures.

Finally, assume that a distribution factor is available; that is, the coefficient $l$ is a function of some observable variable $z$. Then identification obtains within the general set. The trick is that the separable utilities computed from the functions $\phi^{1}$ and $\phi^{2}$ above can no longer be considered as solutions, because the second utility explicitly depends on $l$ and, hence, on $z$-which contradicts the definition of a distribution factor. In other words, the identifying assumption is precisely that the distribution factor $z$ has no direct impact on preferences and matters only through the Pareto weight. Among the numerous structural models that generate the reduced form (26), only the initial one, (23), satisfies this property.

\section{F. Application: Household Production}

Finally, we extend our basic model to include household production. ${ }^{17}$ Specifically, we assume that child utility is "produced" using specific expenditures and parental time. The child's welfare is modeled as $u^{K}\left(K, h^{1}, h^{2}\right)$, where $h_{i}^{K}$ denotes the time spent by member $i$ on household production. In particular, the time constraint for member $i$ becomes

$$
L^{i}+l^{i}+h^{i}=1,
$$

where $L^{i}$ denotes leisure, $l^{i}$ market work, and $h^{i}$ household work. Child welfare is a public good, so that individual preferences take the form

$$
U^{i}\left(C^{i}, L^{i}, u^{K}\left(K, h^{1}, h^{2}\right)\right) \text {. }
$$

Since the outcome of the production is the child's utility, it is not observable and is defined only up to an increasing transformation. It follows that only the functions

$$
\varphi^{1}\left(K, h^{1}, h^{2}\right)=\frac{\partial u^{K} / \partial h^{1}}{\partial u^{K} / \partial K}
$$

and

$$
\varphi^{2}\left(K, h^{1}, h^{2}\right)=\frac{\partial u^{K} / \partial h^{2}}{\partial u^{K} / \partial K}
$$

are identifiable (at best).

Clearly, identifiability of such a structure depends on the type of data that are available. Without time use data, one cannot identify household

\footnotetext{
${ }^{17}$ See also Chiappori (1997) for identification results with marketable household goods and a discussion of identification issues with incomplete markets.
} 
production since neither the inputs nor the output is observed. Now suppose that time use data are available; that is, $h^{1}$ and $h^{2}$ are known functions of $\left(w_{1}, w_{2}, Y, z\right)$. Then productive efficiency requires

$$
\begin{aligned}
& \varphi^{1}\left(K, h^{1}, h^{2}\right)=w_{1}, \\
& \varphi^{2}\left(K, h^{1}, h^{2}\right)=w_{2} .
\end{aligned}
$$

One can then prove the following results: ${ }^{18}$

1. Equations (29) are sufficient to identify the $\varphi^{i}$ over any convex set over which the determinant $D_{\left(w_{1}, w_{2}, Y\right)}\left(h^{1}, h^{2}, K\right)$ does not vanish.

2. Additional, overidentifying predictions can be derived.

3. If these conditions are satisfied, then $u^{K}$ is known up to an increasing transform. By the remark above, without loss of generality we can arbitrarily choose this transform (i.e., a particular cardinalization of $\left.u^{K}\right)$; then $u^{K}$ is known as a function of $\left(K, h^{1}, h^{2}\right)$. Since $K, h^{1}$, and $h^{2}$ are themselves known functions of $\left(w_{1}, w_{2}, Y, z\right), u^{K}$ is ultimately known as a function of $\left(w_{1}, w_{2}, Y, z\right)$. Then proposition 2 applies (replacing $K\left(w_{1}, w_{2}, Y, z\right)$ with $\left.u^{K}\left(w_{1}, w_{2}, Y, z\right)\right)$, and we conclude that the structure is identifiable.

\section{G. Taking the Model to the Data}

The development of our results has had empirical analysis in mind. Indeed the identifiability results relate to the type of data one may have available such as the U.K. Family Expenditure Survey or the U.S. Consumer Expenditure Survey. These surveys include information on household composition, labor supply of individual members, and household consumption broken down by very detailed categories. We require information on expenditures on children, such as food, clothing, education, and so forth, which are not always available but can clearly be collected on the basis of diaries. For identifying models of household production, we would also require a survey in which household members keep a time use diary such as in the American Time Use Survey published by the Bureau of Labor Statistics or other similar surveys listed in the bureau's Web site (http://www.bls.gov/tus/home.htm\#overview). The time use data would need to be combined with expenditure data, which is feasible and can be justified in terms of the insights that can be gained for public policies.

However, any empirical analysis would require a stochastic specification and of course additional identification results in the presence of

\footnotetext{
${ }^{18} \mathrm{~A}$ complete statement and precise proofs are provided in the working paper version of the article (IFS working paper no. WP0208), available at http://www.ifs.org.uk/wps/ wp0208.pdf.
} 
such randomness. Typically we would allow some of the parameters in the utility functions of the individual to be random, in which case we would end up generally with a model that includes nonseparable errors. This is a well-known problem in empirical demand and labor economics.

General identification results are not possible here, but we can illustrate the issues given our Cobb-Douglas example earlier. In the utility functions (12), we could allow the parameters $\alpha_{1}, \alpha_{2}$ and $\delta_{1}, \delta_{2}$ to be random. Although this seems excessive, note that it is plausible to treat the members of the household in a symmetric way. Moreover, the randomness in the parameters $\delta_{1}$ and $\delta_{2}$ ensures that the expenditure on children is not a deterministic function given observables. This specification would imply nonseparable errors in the two observable labor supply equations and the equation for expenditures on children, which is common in structural models other than in the simplest of cases. Identification and estimation with exogenous wages and no corner solutions are straightforward given suitable distributional assumptions on the errors. With endogenous wages, we would need to specify a joint model of wages, labor supply, and expenditures on children, and we could then use standard maximum likelihood methods for estimation. The hardest issue one would encounter in an empirical implementation would be the existence of corner solutions in labor supply, since censoring can lead to serious identifiability issues both from the point of view of the theoretical collective model and from the point of view of the econometric model. Finally, note that one can construe specifications with much simpler stochastic specifications, in which errors are additive as in Blundell et al. (2001).

\section{Conclusion}

It is now becoming widely accepted that in order to analyze the way in which resources are shared within a household, we need to model the household as a collective of individuals rather than as one individual unit. This framework can address issues such as targeting of benefits or distribution of income and consumption within the household. In addition to the clear welfare and policy implications of the collective model, it may also hold the key to why the restrictions from utility theory are often rejected when the unitary model is applied to multimember households. Indeed recent evidence suggests that this may well be the case (Browning and Chiappori 1998; Blundell et al. 2001).

In this paper we extend the Chiappori (1992) framework to include expenditures on public goods, which we like to think of as expenditures on children. We derive the welfare implications of such a model and show that it offers important insights into the issue of targeting. This is uppermost in the policy agenda both in developed and in developing 
countries since governments are particularly concerned about delivering benefits to children, such as schooling or nutrition subsidies. We show that a shift in the Pareto weight toward member 1, say, will always increase member 1's private consumption. It will also increase the demand for the public good if and only if the marginal willingness to pay of member 1 is more sensitive to increases in private consumption than that of member 2. The result emphasizes that basing policy on presumptions about the level of marginal willingness to pay for the public good (e.g., children) is wrong.

The critical parameters for such a policy are an empirical question, and that is precisely why our identifiability theorems are of central importance for the empirical analysis of policies that are supposed to affect the distribution of welfare within a household. We prove identifiability of the structure (which consists of the preferences of each adult in the couple over leisure, consumption, and expenditure on the public good and the Pareto weights) from data that are typically observable in practice, namely, labor supply of individual members, aggregate household expenditure, and expenditure on the public good.

Identifiability obtains under two different conditions. The structure is identifiable if a variable affecting the Pareto weights but not preferences is available; we call this a distribution factor since it affects the distribution of power in the household. Alternatively if a distribution factor is not available, the structure is still identifiable if preferences for consumption and leisure of each household member are weakly separable from expenditures on public goods. Interestingly, we show that separability has implications for the reduced-form labor supply functions that can be checked; this can provide the basis for an empirical test even without the availability of a distribution factor and allows the validity of the identification strategy to be examined. Even so, the conclusion can be that the structure is inconsistent with separable preferences and hence not identifiable without a distribution factor.

Finally, we develop identifiability results for the case in which the public good is an input to household production together with time for each individual. Again we show that the model is identifiable, under the assumption of productive efficiency, as long as time use data are available detailing the time inputs that go into household production (as opposed to pure leisure). Of course the measurement problems here can be severe, but this discussion points to both the importance of collecting data on time use and the importance of distinguishing expenditure on private and public goods.

\section{Appendix}

Lemma and Proof on Which the Separability Test Is Based

Lемма 2. Let $f$ and $g$ be some arbitrary twice continuously differentiable 
functions of $(x, y, z)$. Assume that there exist two functions $a$ and $b$ such that

$$
a(x, f(x, y, z))+b(y, g(x, y, z))=z
$$

for all $(x, y, z)$. Then for any point $(\bar{x}, \bar{y}, \bar{z})$ such that $\partial f / \partial z(\bar{x}, \bar{y}, \bar{z}) \neq 0$, the functions $f$ and $g$ must satisfy, in an open neighborhood of $(\bar{x}, \bar{y}, \bar{z})$, either one or two PDEs.

Proof. Equation (R) implies that

$$
-\frac{f_{y}}{f_{z}}=\frac{b_{y}+b_{g} g_{y}}{1-b_{g} g_{z}},
$$

where $h_{a}$ denotes the partial $\partial h / \partial a$. Hence

$$
\begin{aligned}
b_{y} & =-\frac{f_{y}}{f_{z}}+b_{g}\left(\frac{f_{y}}{f_{z}} g_{z}-g_{y}\right) \\
& =u+b_{g} v,
\end{aligned}
$$

where the functions $u$ and $v$ are defined by $u=-\left(f_{y} / f_{z}\right)$ and $v=\left(f_{y} / f_{z}\right) g_{z}-g_{y}$; note that these functions depend only on $f$ and $g$, so that a condition involving these functions is a condition on $f$ and $g$.

Differentiating with respect to $x$ and $z$, we get

$$
\begin{aligned}
& b_{y g} g_{x}=u_{x}+b_{g g} g_{x} v+b_{g} v_{x}, \\
& b_{y g} g_{z}=u_{z}+b_{g g} g_{z} v+b_{g} v_{z}
\end{aligned}
$$

hence

$$
g_{x} u_{z}-g_{z} u_{x}=\left(g_{z} v_{x}-g_{x} v_{z}\right) b_{g} .
$$

Two cases can be distinguished:

1. In the nongeneric case in which $g_{z} v_{x}-g_{x} v_{z}=0$, it must be the case that $g_{x} u_{z}-g_{z} u_{x}=0$, which gives a PDE.

2. In the alternative, general, case in which $g_{z} v_{x}-g_{x} v_{z} \neq 0$,

$$
b_{g}=\frac{g_{x} u_{z}-g_{z} u_{x}}{g_{z} v_{x}-g_{x} v_{z}} .
$$

The right-hand-side expression must be expressed as a function of $y$ and $g$ only, which gives a first PDE, namely,

$$
g_{z} \frac{\partial}{\partial x}\left(\frac{g_{x} u_{z}-g_{z} u_{x}}{g_{z} v_{x}-g_{x} v_{z}}\right)-g_{x} \frac{\partial}{\partial z}\left(\frac{g_{x} u_{z}-g_{z} u_{x}}{g_{z} v_{x}-g_{x} v_{z}}\right)=0 .
$$

In addition, we have that

$$
b_{y}=u+\frac{g_{x} u_{z}-g_{z} u_{x}}{g_{z} v_{x}-g_{x} v_{z}} v,
$$

and the two partials of $b$ must satisfy the standard cross-derivative restrictions, which provides a second PDE. 


\section{References}

Blundell, Richard W., Pierre-André Chiappori, Thierry Magnac, and Costas Meghir. 2001. "Collective Labor Supply and Household Production." Discussion paper, Inst. Fiscal Studies, London.

Bourguignon, François. 1999. "The Cost of Children: May the Collective Approach to Household Behavior Help?" J. Population Econ. 12 (November): 503-21.

Bourguignon, François, Martin Browning, and Pierre-André Chiappori. 1995. “The Collective Approach to Household Behavior." Working Paper no. 95-04, DELTA, Paris.

Browning, Martin, François Bourguignon, Pierre-André Chiappori, and Valérie Lechene. 1994. "Income and Outcomes: A Structural Model of Intrahousehold Allocation." J.P.E. 102 (December): 1067-96.

Browning, Martin, and Pierre-André Chiappori. 1998. "Efficient Intra-household Allocations: A General Characterization and Empirical Tests." Econometrica 66 (November): 1241-78.

Chiappori, Pierre-André. 1988. "Rational Household Labor Supply." Econometrica 56 (January): 63-90.

. 1992. "Collective Labor Supply and Welfare." J.P.E. 100 (June): 437-67. 1997. "Introducing Household Production in Collective Models of Labor Supply." J.P.E. 105 (February): 191-209.

Chiappori, Pierre-André, and Ivar Ekeland. 2003. "The Micro Economics of Group Behavior: General Characterization.” Manuscript, Univ. Chicago.

. 2004. "The Micro Economics of Group Behavior: Identification." Manuscript, Univ. Chicago.

Chiappori, Pierre-André, Bernard Fortin, and Guy Lacroix. 2002. "Marriage Market, Divorce Legislation, and Household Labor Supply." J.P.E. 110 (February): $37-72$.

Donni, Olivier. 2000. "Essais sur les modèles collectifs de comportement du ménage." PhD diss., l'Ecole des Hautes Etudes en Sciences Sociales, Paris.

Duflo, Esther. 2003. "Grandmothers and Granddaughters: Old-Age Pensions and Intrahousehold Allocation in South Africa." World Bank Econ. Rev. 17 (1): $1-25$.

Fong, Yuk-fai, and Junsen Zhang. 2001. "The Identification of Unobservable Independent and Spousal Leisure.” J.P.E. 109 (February): 191-202.

Fortin, Bernard, and Guy Lacroix. 1997. "A Test of the Unitary and Collective Models of Household Labour Supply.” Econ. J. 107 (July): 933-55.

Galasso, Emanuela. 1999. "Intrahousehold Allocation and Child Labor in Indonesia." Manuscript, Boston Coll.

Koopmans, Tjalling C. 1949. "Identification Problems in Economic Model Construction." Econometrica 17 (April): 125-44.

Lundberg, Shelly J., Robert A. Pollak, and Terence J. Wales. 1997. "Do Husbands and Wives Pool Their Resources? Evidence from the United Kingdom Child Benefit." J. Human Resources 32 (Summer): 463-80.

McElroy, Marjorie B. 1990. "The Empirical Content of Nash-Bargained Household Behavior." J. Human Resources 25 (Fall): 559-83.

Phipps, Shelley A., and Peter S. Burton. 1998. "What's Mine Is Yours? The Influence of Male and Female Incomes on Patterns of Household Expenditure." Economica 65 (November): 599-613.

Rubalcava, Luis, and Duncan Thomas. 2000. "Family Bargaining and Welfare." Working paper (July), Univ. California, Los Angeles. 
Schultz, T. Paul. 1990. "Testing the Neoclassical Model of Family Labor Supply and Fertility." J. Human Resources 25 (Fall): 599-634.

Thomas, Duncan. 1990. "Intra-household Resource Allocation: An Inferential Approach." J. Human Resources 25 (Fall): 635-64.

Thomas, Duncan, Dante Contreras, and Elizabeth Frankenberg. 1997. "Child Health and the Distribution of Household Resources at Marriage." Manuscript, RAND Corp., Santa Monica, CA; Univ. California, Los Angeles. 